Harol do José de Matos 1

Nádia Duppre 2

Maria Fernanda Sardella Alvim 2

Leila Maria MachadoVieira 2

Euzenir Nunes Sarno 2

Cláudio José Struchiner 3

\section{Epidemiologia da hanseníase em coorte de contatos intradomiciliares no Rio de J aneiro} (1987-1991)

\author{
Leprosy epidemiology in a cohort of household \\ contacts in Rio de J aneiro (1987-1991)
}

1 Departamento de Informática Médica, Faculdade de Ciências Médicas, Universidade do Estado do Rio de Janeiro. Av. Prof. Manoel de Abreu 148 20 andar, Rio de Janeiro, RJ 20560-000, Brasil.

2 Setor de Hanseníase Instituto Oswaldo Cruz, Fundação Oswaldo Cruz. Av. Brasil 4365 Rio de Janeiro, RJ 21045-900, Brasil.

3 Programa de Computação Científica, Departamento de Endemias "Samuel Pessoa", Escola Nacional de Saúde Pública, Fundação Oswaldo Cruz. Rua Leopoldo Bulhões 1480, Rio de Janeiro, RJ 21041-210, Brasil.
A bstract This study ai med to identify factors influencing the devel opment of Ieprosy (Hansen's disease) in household contacts. A dynamic cohort was analyzed from 1987 to 1991 at the Hansen's Disease Department of the Oswal do Cruz Foundation in Rio de Janeiro. The incidence rate was 0.01694 person-years of follow-up. Nevertheless, for subjects at the end of the first year of followup the incidence rate was 0.06385 (end of second year, 0.03299; end of third year, 0.02370; end of fourth year, 0.018622; and end of observation period, 0.01694). A stepwise multivariate logistic regression model was proposed to study the risk of devel oping leprosy, including co-prevalent cases, totaling 758 contacts. In the final model, the risk was associated with a negative Mitsuda skin test $(\mathrm{OR}=3.093 ; \mathrm{Cl} 95 \%=1.735-5.514)$, prior $B C G$ vaccination $(\mathrm{OR}=0.3802 ; \mathrm{Cl} 95 \%=$ 0.2151-0.66719), and multibacillary primary clinical form (OR =2.547; Cl 95\%=1.249-5.192). The results showed that both multibacillary leprosy and specific immune status are si gnificant indicators for developing the disease in a cohort of household contacts.

Key words Leprosy; Logistic Regression; Incidence; Cohort Studies; Epidemiology

Resumo Este trabalho foi desenvolvido para estudar os fatores que influem no adoeci mento dos contatos intradomiciliares de hanseníase. Foi estabel eci da uma coorte de contatos intradomiciliares de hanseníase no setor de Hanseníase da Fiocruz, no Rio de Janeiro. Entre 1987 e 1991, em que foram acompanhados 670 contatos saudáveis, a taxa de inci dência por pessoa-ano de followup foi de 0,01694. Porém, houve variação com o tempo de acompanhamento de cada indi víduo, sendo de 0,06385 ao final do primeiro ano, de 0,03299 ao final do segundo, 0,02370 ao final do terceiro, 0,018622 a final do quarto e 0,01694 ao final do período. Foi especificado um modelo de regressão logística para os indivíduos acompanhados nos primeiros cinco anos, envol vendo 758 contatos, incluindo nessa situação os casos co-prevalentes. O risco de inci dência de hanse níase está relacionado com a negatividade do teste de Mitsuda, com OR de 3,093 (IC95\% =1,7355,514), com a vacinação pelo BCG, OR de 0,3802 (IC95\%=0,2151-0,66719), e com a forma multibacilar do caso-índice, OR de 2,547 (IC95\%=1,249-5,192). Os resultados indicam quea incidência da hanseníase em contatos está associada à forma multibacilar da doença e aos fatores imunes dos contatos.

Palavras-chave Hanseníase; Regressão Logística; Incidência; Estudos de Coortes; Epidemi ologia 


\section{Introdução}

O Brasil inclui-se entre os países de alta endemicidade de hanseníase no mundo. Isto significa que apresenta um coeficiente de prevalência médio superior a um caso por mil habitantes (MS, 1989). Embora, geograficamente, esse coeficiente seja desigual, com áreas de alta, média e baixa prevalência distribuídas em todo o território do país, há uma tendência uniforme de crescimento no número de casos diagnosticados desde os anos 70 até agora. E, atualmente, a hanseníase representa uma das poucas doenças infecciosas com elevação de incidência em nosso país (IBGE, 1995), uma tendência contrária àquela que vem ocorrendo em termos mundiais (OMS, 1998).

Diante desse quadro, medidas de prevenção e controle, além do tratamento eficaz dos casos, são absolutamente necessárias. Enquanto uma vacina efetiva para hanseníase não se encontra disponível, programas de vigilância epidemiológica assumem importância central no controle e prevenção da doença (MS, 1989). Como a transmissão do Mycobacterium leprae ocorre de indivíduo para indivíduo, os contatos intradomiciliares constituem um grupo de risco. Em um estudo recente, Chanteau et al. (1993) determinaram um risco relativo de 30,8 para o aparecimento de hanseníase entre os contatos familiares em comparação com nãocontatos, em um estudo de coorte de 1201 contatos durante dez anos. Um aspecto interessante na análise desse grupo é que mesmo constituindo-se em um grupo de risco, um percentual relativamente pequeno dos contatos tornou-se doente em um período de cinco anos, com taxas de incidência em torno de 1 a $2 \%$, neste estudo.

Em uma revisão da epidemiologia da hanseníase de 1962-1992, Nordeen (1993) conclui que todas as evidências disponíveis indicam claramente que a proporção de pessoas expostas à lepra que adquirem infecção é muito maior do a daquelas observadas com expressão clínica da doença. Entretanto, o que não parece claro, ainda, é se os indivíduos que desenvolvem a doença clínica representam uma proporção constante dos que foram infectados, e, se não, que fatores influenciam esta proporção. De qualquer modo, provavelmente, os fatores que determinam a ocorrência da doença após a infecção são tão ou mais importantes do que aqueles que determinam a ocorrência da infecção após a exposição.

Uma pergunta básica surge então: Quais são os fatores que mais influenciam o aparecimento da doença nos contatos intradomicilia- res? Esses fatores podem ser de naturezas as mais diversas, desde os fatores ligados aos casos-índices, passando por aspectos ambientais, até fatores ligados aos contatos propriamente.

A compreensão desse modelo conceitual poderá abrir um caminho novo para a epidemiologia da hanseníase, como instrumento efetivo de controle e eliminação desta doença. Pois a hanseníase constitui-se em uma das poucas doenças infecciosas em que um modelo animal não pode ser desenvolvido com sucesso, a não ser em situações especiais com tatus (MS, 1989). Além disso, estudos imunológicos em hanseníase têm enfatizado o papel central da interação M. Ieprae macrófago na patogênese da doença (Sampaio et al., 1991). Desta forma, a modelagem matemática e estatística de dados epidemiológicos pode surgir como um instrumento capaz de prever cenários (Anderson \& May, 1991; Massad, 1992), com base nos dados disponíveis, para o comportamento da doença sob várias situações, e desta forma orientar e re-orientar intervenções possíveis na prevenção, tratamento e eliminação da hanseníase em nosso meio.

No setor de Hanseníase do Instituto Oswaldo Cruz, centro de referência em hanseníase no Rio de Janeiro, iniciou-se um programa de acompanhamento de contatos de hanseníase em 1987. Atual mente esta coorte é composta por 2225 contatos. Alguns desses contatos tornaram-se doentes durante este período de dez anos (a uma taxa média de 4,5\%). O objetivo deste trabalho é responder às duas perguntas colocadas acima. Quais os fatores mais importantes para o aparecimento da doença? E, em conseqüência, quais os que mais contribuíram para uma resistência elevada ao aparecimento da doença? Procuraremos medir a importância relativa de fatores demográficos, familiares e individuais para o aparecimento da doença. Para tal, foram estimadas a taxa de incidência da hanseníase na coorte de contatos estudada e, para a estimação do risco de adoecimento, foi proposto um modelo de regressão logística multivariado.

\section{Material e métodos}

A investigação epidemiológica dos contatos

A partir de 1987, foi organizada uma coorte dinâmica de contatos intradomiciliares de hanseníase que se valeu dos casos-índices diagnosticados no Ambulatório Souza Araújo do Setor de Hanseníase da Fundação Oswaldo Cruz do Rio de Janei ro, que é um centro de referên- 
cia regional e nacional do Ministério da Saúde para o diagnóstico e a pesquisa em hanseníase, sediado no Estado do Rio de Janeiro. Por isso, esse ambulatório recebe pacientes encaminhados de várias instituições de saúde do estado, tanto públicas como privadas, merecendo destaque as instituições universitárias. A coorte de contatos conta no momento com 2225 contatos. O período de estudo da nossa análise compreende os contatos registrados entre 1987 e 1991. Estes contatos estão e continuarão sendo acompanhados anualmente, ou até que o próprio indivíduo detecte algum tipo de lesão dermatológica ou apresente alguma queixa.

No exame inicial, além do exame dermatológico tradicional, um exame físico detalhado é realizado, bem como são solicitadas provas imunológicas, como o teste cutâneo de Mitsuda, e dosagem de PGL-1 e $\gamma$-interferon. A partir de 1992, seguindo orientação do Ministério da Saúde, todos os contatos intradomiciliares são vacinados com BCG. Os exames subseqüentes, anuais, consistem de entrevista, exame físico, e coleta de soro. Foi elaborada uma base de dados com informações ambientais, demográficas, familiares e individuais destes contatos, bem como um cadastro detal hado dos casosíndices. A base de dados foi construída com o uso do software Epi Info, versão 6.04b (Dean et al., 1997), como parte de um sistema de informações em hanseníase utilizado no setor. As variáveis incluíram três níveis de análise, como descrito abaixo:

- Família

1) número de pessoas na casa; 2) número de cômodos; 3) nível sócio-econômico; 4) situação ambiental da moradia; 5) origem geográfica da família.

\section{- Os contatos}

1) critério de diagnóstico; 2) tempo do aparecimento da doença; 3) forma clínica do caso-índice; 4) família; 5) idade; 6) sexo; 7) raça; 8) relação com o caso-índice; 9) ocupação; 10) nível educacional; 11) tempo de contato.

- Micro-ambiente biológico

1) tipo clínico do contato doente; 2 ) vacinação com BCG; 3) teste cutâneo com PPD; 4) teste cutâneo com Mitsuda; 5) índice baciloscópico do caso-índice; 6) nível de IgM anti-PGL-1.

O diagnóstico de hanseníase, tanto nos casos-índices, como nos casos secundários derivados da coorte de contatos, segue os critérios da OMS. Estes critérios estão baseados principalmente em sintomas e sinais clínicos. São fáceis de serem avaliados por qualquer profissional da área de saúde, após um curto período de treinamento, desde que haja uma orientação para isso. O que a OMS preconiza para países ou áreas endêmicas é o achado de um dos seguintes sinais cardinais: 1) lesão de pele consistente com hanseníase e acompanhada de perda sensitiva, com ou sem espessamento de nervos; 2) esfregaços de pele positivos para $M$. leprae. No caso dos pacientes e contatos acompanhados no ambulatório Souza Araújo, além desses critérios, todos os casos são submetidos à biópsia (OMS, 1988).

Os casos diagnosticados podem ser classificados com base nas manifestações clínicas e nos resultados do esfregaço da pele. Na classificação baseada nos esfregaços da pele, os pacientes com esfregaços negativos em todas as lesões são agrupados como paucibacilares, enquanto que os que apresentam esfregaços positivos em qual quer localização são chamados de multibacilares. Na prática, a maioria dos serviços utiliza um critério clínico para a classificação. O sistema clínico, para o propósito de tratamento, inclui o uso do número de lesões de pele e nervos envolvidos, com base na classificação em hanseníase Multibacilar (MB) e Paucibacilar (PB) (OMS, 1988). No estudo atual, além dessa classificação, utilizou-se ainda a classificação de Ridley-Joplin (1966), o que é facilitado pelo fato de que em todos os casos há biópsias. Esta classificação agrupa os casos de hanseníase em cinco grupos: LL, BL, BB (Lepromatous, Borderline Lepromatous, Borderline) - que são considerados como multibacilares; e BT, TT (Borderline Tuberculous, Tuberculous, Indeterminada) - que são considerados como paucibacilares, além de uma forma clínica inicial, HI (Indeterminada) que pode evoluir para qualquer das formas anteriores.

O cálculo da taxa de incidência na coorte de contatos

A estimação das taxas foi feita pelo método de densidade de incidência (Kleinbaum, 1982). Neste método, em primeiro lugar é calculado o número de pessoas-ano, a partir da detecção do caso-índice. Neste caso, o período de observação de cada indivíduo foi calculado levandose em conta a data do diagnóstico do caso-índice, e estende-se pelo período em que o indivíduo permanecer na família ou desenvolver doença.

Os cál culos foram baseados na fórmula:

$\mathrm{DI}=\mathrm{I} / \mathrm{PT}$ 
onde, $\mathrm{DI}=$ Densidade de inciência; I = número de casos incidentes; e PT = pessoas-tempo. No caso, pessoas-tempo = pessoas-ano. E o cálculo de PT é dado pela fórmula:

$$
\mathrm{N}
$$$$
\mathrm{PT}=\Sigma \Delta \mathrm{t}_{\mathrm{i}}
$$

onde, $\mathrm{N}=$ Número de pessoas na coorte; $\mathrm{e}$ $\Delta \mathrm{t}=$ intervalo de tempo entre a entrada na coorte e a sua saída (ou adoecimento ou última informação).

Para a estimação dessas taxas, foram incluídos somente os contatos que não apresentavam qualquer evidência de doença no momento do diagnóstico do caso-índice, totalizando 670 contatos nessa situação. E foram estimadas as taxas de incidência pelo período de acompanhamento da coorte entre 1987 e 1991, e também as taxas dentro de cada ano de acompanhamento da coorte. Exemplo: uma taxa para os contatos acompanhados até 1 ano; uma outra para os acompanhados por um período de até 2 anos; e assim até a taxa para o conjunto dos contatos acompanhados por 4 anos ou mais.

Especificação da Modelagem do risco de adoecimento na coorte de contatos usando-se um modelo logístico multivariado passo a passo

Dos contatos acompanhados nos primeiros cinco anos de estudo, alguns contatos intradomiciliares chamados a exame após um diagnóstico confirmado de um caso-índice na família já apresentavam também a doença. São os chamados casos co-prevalentes. Embora esses casos tenham sido excluídos da análise das taxas de incidência, optou-se pela sua inclusão na modelagem do risco pelo modelo de regressão logística, pois a maioria destes exibia uma forma indeterminada, ou paucibacilar de hanseníase. Neste caso, interpretou-se esse contato como tendo adoecido a partir de contágio com o caso-índice, embora não se tenha, com precisão, o tempo de doença e em conseqüência, não se possa estimar, com exatidão, o tempo até o adoecimento.

Para este problema, como a varíavel dependente é uma variável binária, adoecimento ou não, e como em nem todos os casos pode-se estimar o tempo ocorrido para o adoecimento, tudo se passaria como se o tempo estivesse colapsado para o conjunto das observações dos casos co-prevalentes. Por isso, uma abordagem inicial para a modelagem do risco foi o modelo de regressão logística multivariado.

No contexto do estudo epidemiológico da coorte de contatos, são observadas variáveis explanatórias $\mathrm{X}_{1}, \mathrm{X}_{2}, \ldots \mathrm{X}_{\mathrm{k}}$, baseadas no conjunto dos dados observados. Para cada indivíduo, ao final do período de observação foi especificado seu estado de doença, ou 1 se "doente" ou 0 se "não doente". A probabilidade de desenvolvimento da lepra que está sendo modelada pode ser expressa pela probabilidade condicional $P\left(D=1 \mid X_{1}, X_{2}, \ldots, X_{k}\right)$. Esta probabilidade pode ser definida como logística, se a expressão para a probabilidade de desenvolvimento da hanseníase, dadas as variáveis $X_{S}$ for dada por:

$\mathrm{P}\left(\mathrm{D}=1 \mid \mathrm{X}_{1}, \mathrm{X}_{2}, \ldots, \mathrm{X}_{\mathrm{k}}\right)=1 / 1+\mathrm{e}^{-(\alpha+\Sigma \beta \mathrm{X} \mathrm{i})}$ (Hosmer \& Lemeshow, 1989; Kleinbaum, 1994).

Os termos $\alpha$ e $\beta$ i no modelo representam parâmetros estimados com base nos dados obtidos da coorte. Então, para qualquer contato livre de doença, a partir da observação das variáveis $X_{1}$ até $X_{k}$, poderá ser obtida uma probabilidade de que este indivíduo apresentará hanseníase em algum intervalo de tempo definido.

Foram incluídas na especificação incial do modelo de regressão logística as variáveis descritas no item anterior (Material e métodos). $E$, como também foi descrito nessa seção, foram incluídos os casos co-prevalentes, isto é, aqueles casos em que o primeiro exame do contato domiciliar evidenciou também neste a presença de hanseníase em adição ao caso-índice, ou caso primário. Então, aos 670 contatos acompanhados somaram-se 88 casos co-prevalentes, que foram considerados contatos que se tornaram doentes para efeito da aplicação do modelo de regressão logística, devido ao fato de apresentarem formas clínicas de hanseníase paucibacilares em comparação com as formas clínicas multibacilares dos casos-índices em questão. Assim, pelo conhecimento prévio da epidemiologia e das características da transmnissão do M. leprae, adotou-se a estratégia de não sacrificar-se a informação pontencialmente presente nos casos co-prevalentes (ver Resultados para detal hes na distrubição das formas clínicas neste grupo).

Com base nas observações disponíveis no banco de dados, e de acordo com o conhecimento adquirido sobre a infectividade do $M$. leprae, que é transmitido de pessoa a pessoa, com importância das variáveis relacionadas ao micro-ambiente familiar, às características demográficas individuais, e particularmente à situação imunológica do sujeito. Desta forma, o modelo inicial incluiu as seguintes variáveis: variável dependente - (doente: sim/não); variáveis explanatórias: Mitsuda (negativo/ positivo); PPD (contínua); BCG (vacinado/não vacinado); Forma clínica do caso-índice (multibacilar/ paucibacilar); Tempo de convivência (contínua); Idade (contínua); Forma de con- 
vivência (cônjuge/ não cônjuge); cor (branca/ parda/ negra), e sexo (masculino/feminino). Foi adotado o modelo passo a passo, em que uma forma final do modelo é adotada como o meIhor modelo fundamentado da especificação inicial. No procedimento passo a passo, as variáveis que não atingem um nível de significância pré-estabelecido, que na análise atual foi fixado em 95\%, são eliminadas do modelo, sucessivamente, até que o modelo final contenha somente variáveis estatisticamente significativas. O modelo foi desenvolvido com o programa estatístico Egret (Statistics and Epidemiology Research Corporation/ Cytel Software Corporation, 1994).

\section{Resultados}

Estimação das taxas de incidência de hanseníase nos primeiros cinco anos da coorte

Entre 1987 e 1991, 670 contatos intradomiciliares que não apresentaram qualquer evidência de hanseníase no exame incial foram acompanhados anualmente, ou até que algum sinal de doença fosse detectado. As observações individuais variaram entre 2 meses e 4 anos e 2 meses no período do estudo. Os 670 contatos foram derivados de 152 casos primários, dos quais $72,2 \%$ apresentavam forma clínica multibacilar (BB/BL/ LL, na classificação de Ridley-Jopling), enquanto $27,8 \%$ apresentavam forma clínica paucibacilar. Durante o período de follow-up, entre os 670 contatos acompanhados em pe- ríodos variáveis de tempo, apareceram 23 casos secundários: 2 (8,69\%) na forma clínica multibacilar (BB/BL), 11 (47,83\%) na forma clínica paucibacilar (BT/TT), e $10(43,48 \%)$ na forma clínica indeterminada da hanseníase.

Os resultados das taxas de incidência apresentados (Tabela 1 e Figura 1) estão relacionados ao conceito de pessoas-ano de follow-up de contatos a partir da detecção do caso primário. Isto é, referem-se ao período de observação de cada indivíduo calculado a partir da entrada de cada indivíduo na família ou da data de detecção do caso primário, o que for mais tardio, durante a permanência do indivíduo na família ou o aparecimento da doença.

Pode-se observar que, à medida em que os pacientes permanecem na coorte, a incidência cai em função do tempo de follow-up. É interessante observar que o aspecto desta curva de taxa de incidência decrescente assemelha-se ao aspecto de uma curva de função de intensidade de incidência (ou hazard de padrão de Weibull decrescente, um modelo paramétrico utilizado em análise de sobrevida).

Modelagem do risco de adoecimento na coorte de contatos usando-se um modelo logístico multivariado passo a passo

A Tabela 2 apresenta as variáveis incluídas no modelo inicial utilizado no processo de análise passo a passo. É interessante observar o fato da predominância das formas multibacilares dos casos primários dos contatos da coorte, chegando a 72,2\% das formas clínicas dos casos-

\begin{tabular}{|c|c|c|c|c|c|}
\hline & até 1 ano & $1-2$ anos & $\begin{array}{l}\text { Ano da coort } \\
2-3 \text { anos }\end{array}$ & 3-4 anos & $>4$ anos \\
\hline No de contatos na coorte no período específico & 81 & 288 & 160 & 99 & 42 \\
\hline No de contatos acumulado na coorte até o fim do período & 81 & 369 & 529 & 628 & 670 \\
\hline № de casos secundários na coorte no perío do específico & 4 & 11 & 5 & 2 & 1 \\
\hline $\begin{array}{l}\text { № de casos secundários acumulado na coorte } \\
\text { até o fim do período }\end{array}$ & 4 & 15 & 20 & 22 & 23 \\
\hline Taxa de ataque bruta acumulada até o fim do período & 0,05 & 0,04 & 0,04 & 0,04 & 0,03 \\
\hline № de pessoas-ano até o fim do período & 62,64682 & 333,43437 & 843,88185 & 1181,39834 & 1357,73317 \\
\hline $\begin{array}{l}\text { Taxa de incidência (por pessoa-ano de follow-up) na coorte } \\
\text { até o fim do período }\end{array}$ & 0,06385 & 0,03299 & 0,02370 & 0,018622 & 0,01694 \\
\hline
\end{tabular}

Nota: O cálculo das taxas de incidência foi realizado de acordo com a fórmula: DI = I/PT, onde DI é a densidade de incidência, apresentada na última coluna, o número de pessoas-tempo (PT) é o resultado do cálculo de pessoas-ano, e é apresentado na penúltima coluna, e I, é o número de casos secundários, acumulado na coorte até o fim de cada período de observação, em anos, apresentado na coluna 5. 
Figura 1

Densidade de incidência em pessoas-ano de follow-up.

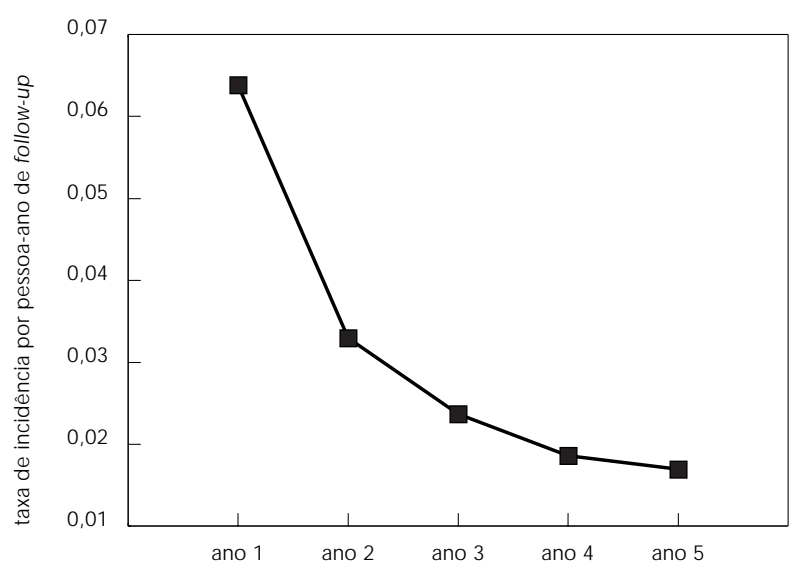

O bs: O s valores plotados para taxa de incidência são os valores da taxa de incidência acumulada, i. e., o primeiro valor plotado foi estimado até o final do primeiro ano. ca fosse retirada do modelo. Pelos resultados do diagnóstico, de um modo geral, foram observados poucos casos com grande variação nos parâmetros do modelo final caso fossem retirados. Os parâmetros mais sensíveis à variação foram os associados à variável explanatória do resultado do teste Mitsuda.

\section{Discussão}

Apesar de haver poucos relatos na literatura sobre estudos de coortes em contatos de hanseníase, eles têm surgido mais recentemente, enfatizando o papel relevante do contato intradomiciliar na epidemiologia da hanseníase, e em especial têm chamado a atenção para possibilidades estratégicas para o controle e a eliminação da doença, o que tem sido reiterado como um objetivo básico da Organização Mundial da Saúde (OMS, 1998).

Considerando-se o estudo de Ranade et al . (1995), em que foram apresentados resultados sobre taxas de incidência de hanseníase em famílias em uma área endêmica na Índia, constata-se uma incidência bem maior de hanseníase entre os contatos no estudo presente, em comparação com o estudo indiano. A taxa global de incidência apresentada naquele estudo foi de 4,46 por 1000 pessoas ano de risco, no período entre 1952 e 1986. No nosso estudo, a taxa global de incidência foi de 16,94 por 1000 pessoas-ano de follow-up no período entre 1987 e 1991. Uma diferença básica na interpretação das diferentes taxas está no fato de que no estudo de Ranade as taxas foram calculadas em pessoas-ano de risco, e não de follow-up. Este autor utiliza este conceito baseado em Frost (1933), considerando cada ano de vida de cada indivíduo como uma unidade. Desta forma o cálculo de pessoas ano de risco por período de contato é baseado no período de contato, que é calculado a partir da data de detecção do caso primário até o final do período de atividade da doença do caso primário, ou até que o contato saudável deixe de morar na família, o que for menor. Entretanto, nesse estudo não fica claro o critério para o cálculo do período de risco. Algumas dificuldades práticas para a aplicação deste conceito residem no fato de se estabelecer com certeza o tempo de doença, e em muitos casos há dificuldades para a precisão do período de permanência de alguns indivíduos na família. Além disso, outra dificuldade adicional está na determinação do fim do risco para os contatos após o início do tratamento do caso primário. Mesmo considerando-se o atual esquema de poliquimioterapia preconizado pela 
OMS, não há consenso entre os especialistas para um critério de tempo em que um indivíduo possa ser considerado como curado. $O$ esquema tem a duração clássica de dois anos, mas alguns especialistas consideram que em 1 ou 2 meses o indivíduo doente já possa estar curado. Em nossa opinião, todas estas questões representam dificuldades para a aplicação do conceito correto de pessoas-ano de risco, por mais atraente que ele seja. Por isso, parece-nos que o conceito de pessoas-ano de follow-up possa traduzir o fenômeno da transmissibilidade da hanseníase, mesmo de uma forma indireta.

Não é, portanto, surpreendente que os dados relativos à taxa de incidência dentre os contatos caiam anualmente nos anos de follow-up seqüenciais. Isto porque, uma vez tratado o indivíduo, a probabilidade de adoecimento entre os seus contatos intra-domicial res tende a cair. No entanto, embora mais raros, os casos secundários continuam a ocorrer (Figura 1). Isso pode ser devido a um longo período de incubação da hanseníase, após longos períodos de exposição, representando o fato de que um determinado indivíduo possa estar infectado, mas não doente, caracterizando a figura de portador, como parece sugerir Nordeen, em sua revisão da epidemiologia da hanseníase. Uma segunda interpretação seria a de que a fonte de infecção nestes casos secundários, possivelmente os mais tardios (com mais de 2 anos de follow-up, por exemplo), pudesse ser outra. Ou alguém de fora da família, ou atéainda um portador de hanseníase assintomático na família. Entretanto, como Nordeen bem explicita, o problema é que ainda não se dispõe de um indicador eficaz do estado de infecção, sem manifestações clínicas da doença. As conclusões de Chanteau et al. (1993) também apontam no sentido de que a PGL-1 não é um indicador seguro de infecção subclínica e que apresenta um valor preditivo positivo baixo para o desenvolvimento futuro de hanseníase. $\mathrm{E}$, embora comente da necessidade de futuros testes para a detecção de infecção pelo M. le prae, ela se apresenta cética em relação à utilidade deles em programas de controle da doença. No entanto, parece-nos que a possibilidade de existirem portadores assintomáticos na hanseníase, e que estes portadores possam ser fonte de infecção para contatos intra-domiciliares, não pode ser facilmente descartada, pelo fato de não possuirmos um indicador seguro. Pelo contrário, parece-nos fundamental investigar essas possibilidades no contexto mesmo de medidas de controle.

Os nossos resultados sobre as taxas de incidência de hanseníase entre os contatos intra-

\begin{tabular}{|c|c|}
\hline \multicolumn{2}{|c|}{$\begin{array}{l}\text { Perfil das variáveis incluídas na especificação inicial } \\
\text { do modelo de regressão logística passo a passo. }\end{array}$} \\
\hline \multicolumn{2}{|l|}{ Variáveis } \\
\hline \multicolumn{2}{|c|}{ Reação de Mitsuda } \\
\hline Negativo & $19,2 \%$ \\
\hline Positivo & $80,8 \%$ \\
\hline \multicolumn{2}{|l|}{$\mathrm{BCG}$} \\
\hline Vacinado & $50,5 \%$ \\
\hline Não-vacinado & $49,5 \%$ \\
\hline \multicolumn{2}{|l|}{ PPD (mm) } \\
\hline Média & 9,436 \\
\hline Desvio-padrão & 7,137 \\
\hline \multicolumn{2}{|c|}{ Forma de convivência } \\
\hline Cônjuge & $16,6 \%$ \\
\hline Não-cônjuge & $83,4 \%$ \\
\hline \multicolumn{2}{|c|}{ Tempo de convivência (anos) } \\
\hline Média & 18,003 \\
\hline Desvio-padrão & 11,297 \\
\hline \multicolumn{2}{|l|}{ Idade (anos) } \\
\hline Média & 28,024 \\
\hline Desvio-padrão & 16,086 \\
\hline \multicolumn{2}{|l|}{ Cor } \\
\hline Branca & $69,4 \%$ \\
\hline Parda & $21,6 \%$ \\
\hline Negra & $9,0 \%$ \\
\hline \multicolumn{2}{|c|}{ Forma clínica do caso primário } \\
\hline Multibacilar & $71,2 \%$ \\
\hline Paucibacilar & $28,8 \%$ \\
\hline \multicolumn{2}{|l|}{ Sexo } \\
\hline Feminino & $58,0 \%$ \\
\hline Masculino & $42,0 \%$ \\
\hline
\end{tabular}

domiciliares de hanseníase parecem indicar no sentido da derrubada de um mito, partilhado por muitos, de que a vigilância de contatos não é uma medida importante em um contexto de área de alta endemicidade (Chanteau et al., 1993). Se considerarmos as taxas de incidência na população geral $(1,42$ por 1000 , a taxa de incidência média para o Brasil em 1987 - MS, 1989), chegaremos à conclusão de que as taxas nos nossos contatos são pelo menos 12 vezes maiores, o que indica um RR de 12 . O estudo de Chanteau et al. (1993) mostrou um RR de 30 , também em um contexto de alta endemicidade. Assim, parece-nos razoável admitir que a 
Especificação do modelo final de regressão logística passo a passo.

\begin{tabular}{lcccc}
\hline Variável & Coeficiente & Valor p & Odds Ratio & $\begin{array}{c}\text { Intervalo de } \\
\text { Confiança (95\%) }\end{array}$ \\
\hline \% GM & $-2,720$ & $<0,001$ & 0,658 & $0,3273-0,1325$ \\
Mitsuda & 1,129 & $<0,001$ & 3,093 & $1,735-5,514$ \\
BCG & $-0,961$ & $<0,001$ & 0,3802 & $0,2151-0,6719$ \\
Forma clínica do caso primário & 0,9348 & 0,010 & 2,547 & $1,249-5,192$ \\
\hline
\end{tabular}

Obs: o modelo foi especificado para a variável dependente Doente, e são apresentados ainda os intervalos de confiança para cada parâmetro estimado.

vigilância de contatos, embora uma atividade árdua e difícil pode trazer resultados palpáveis para não só o controle, como a eliminação da hanseníase em um contexto de alta endemicidade.

Por outro lado, a generalização dos dados de incidência apresentados no nosso estudo deve ser considerada de modo cuidadoso. Em primeiro lugar, porque deve ser considerada a possibilidade de um viés de seleção na composição de nossa coorte, pois a demanda ao serviço de referência ocorre de duas formas: a espontânea e a referenciada. Desta maneira, os casos-índices formam um grupo que não foi selecionado com um critério aleatório com base na população de hansenianos do município. Em segundo lugar, talvez como decorrência de um referenciamento preferencial de casos mais graves, há um nítido predomínio de casos multibacilares entre os casos-índices (71,2\%). Levando-se em conta essas ponderações, poderíamos admitir que as taxas de incidência em nossa coorte possam estar superestimadas, quando comparadas a outras condições de análise, como é discutido acima em comparação ao estudo de Ranade, na Índia.

Um dos objetivos centrais do presente estudo foi a tentativa de estabelecer entre os contatos um subconjunto com maior probabilidade de adquirir hanseníase, ou seja, um subconjunto que representasse um grupo de risco para o adoecimento. Neste sentido, a modelagem pareceu-nos um instrumento útil, e já tão utilizado em outras doenças, como a Aids, por exemplo (Nowak, 1991; Grossman, 1993; Mosier, 1994). Infelizmente, estudos de coorte de contatos em hanseníase são escassos, e praticamente nenhum deles utiliza modelagem, com poucas exceções (van Brackel, 1994). Os modelos que têm sido utilizados em séries históricas são curvas de ajustamento ou tendência. Embora uma estratégia de modelagem inicialmente mais atraente para a coorte de contatos apresentada no presente estudo seja o uso de modelos de sobrevivência, esbarra-se no fato de que no período analisado, 87-91, houve relativamente poucos casos novos (23), dentre os 670 contatos acompanhados. Por outro lado, foram detectados mais 88 casos co-prevalentes, entre os contatos. Como não há informação substantiva em termos temporais para esses casos, optou-se pelo uso de um modelo de regressão logística multivariado para a indicação de possíveis fatores de risco dentre os contatos, mesmo minimizando-se a informação temporal disponível para os 670 contatos acompanhados, incluindo-se aí os 23 que adoeceram.

Os resultados do modelo de regressão logística indicam que os contatos com Mitsuda inicial negativo, não vacinados com BCG e que têm casos primários com formas clínicas multibacilares de hanseníase, constituem um grupo de risco para o adoecimento. O resultado inicial do teste de Mitsuda representa um conjunto de subvariáveis que têm imporância fundamental na susceptibilidade individual para se adquirir hanseníase. Esse subconjunto inclui desde a influência do HLA sobre a resposta imune à hanseníase (Neeloo, 1997), até a efetivação desta resposta na produção de $\gamma$-Interferon (Sampaio et al., 1991). Ou seja, embora o Mitsuda seja um teste relativamente grosseiro, que é determinado por múltiplos subfatores, e dinâmico, que pode variar no tempo e em função de uma série de variáveis individuais e clínicas, parece constituir um indicador de risco entre os contatos intradomiciliares de hanseníase, independente da vacinação pelo BCG, e da forma clínica de hanseníase do caso primário.

Quanto ao BCG, Fine, em uma série de trabalhos (Fine, 1997), demosntrou sua eficácia como agente protetor contra a hanseníase. Inclusive, entre os contatos intradomiciliares de hanseníase do presente trabalho, um dos autores (Alvim, 1993) confirmou o papel protetor do BCG, em um estudo caso-controle. 
A terceira variável de risco, a forma clínica multibacilar, é de interpretação esperada, a partir do conhecimento da forma de transmissão individual de hanseníase (Nordeen, 1993; vanBeers, 1994). O que o modelo parece indicar, no entanto, é que esse não é um fator suficiente para o aparecimento da doença hanseníase, e que os fatores imunológicos e individuais têm tanta ou maior importância no desenvolvimento da doença. Se, no entanto, uma grande parte dos contatos intradomiciliares de casos primários multibacilares, ou até a totalidade, se infecta mas não desenvolve a doença, permanece uma questão aberta. É provável que a asso- ciação da forma clínica multibacilar com o adoecimento, mostrada no modelo (Tabela 3), esteja relacionada com um maior índice de contatos infectados neste grupo em comparação com os contatos de pacientes paucibacilares, e que este maior índice está relacionado com uma maior probabilidade de adoecimento. Mais uma vez, deparamo-nos com a possibilidade de que a ocorrência de portadores assintomáticos venha a trazer uma explicação para a transmissibilidade da doença, e que a sua detecção venha a ser uma das ferramentas mais úteis no futuro para o controle da hanseníase, particularmente entre os contatos intradomiciliares.

\section{Agradecimentos}

Este estudo foi parcialmente financiado pela OMS e pelo CNPq. A Dra. Leila Maria Machado Vieira é pesquisadora visitante da FAPERJ. O Dr. Cláudio José Struchiner foi parcialmente financiado pelo CNPq e PRONEX/FINEP.

\section{Referências}

ALVIM, M. F. S., 1993. Efeito Protetor do BCG na Hanseníase. Um Estudo Caso-controle. Dissertação de Mestrado, Rio de Janeiro: Instituto de Medicina Social, Universidade do Estado do Rio de Janeiro.

ANDERSON, R. M . \& MAY, R. M., 1991. Infections Diseases of Humans. Dynamics and Control. London: Oxford University Press.

CHANTEAU, S.; GLAZIOU, P.; PLICHART, C.; LUQUIAUD, P.; PLICHART, R.; FAUCHER, J. F. \& CARTEL, J. L., 1993. Low predictive value of PGL-I serology for the early diagnosis of leprosy in family contacts: Results of a 10-year prospective field study in French Polynesia. International Journal of Leprosy and Other Mycobacteria Diseases, 61: 533-541.

DEAN, A. G.; DEAN, J. A.; BURTON, A. H. \& DICKER, R. C., 1997. Epi-Info, Version 6.04b: A Word Processing, Database, and Statistics Program for Epidemiology on Micro-computers. Atlanta: Centers for Disease Control.

FINE, P. E.; STERNE, J. A.; PÖNNIGHAUS, J. M.; BLISS, L.; SAUI, J.; CHIHANA, A.; MUNTHALI, M. \& WARNDORFF, D. K., 1997. Household and dwelling contact as risk factors for leprosy in northern Malawi. American Journal of Epidemiology, 146: 91-102.

FROST, W. H., 1933. Risk of persons in family contact with pulmonary tuberculosis. American Journal of Health, 23:426-432.

GROSSMAN, Z.; BENTWICH, Z. \& HERBERMAN, R. B., 1993. From HIV infection to AIDS: Are the manifestations of Effective immune resistance misinterpreted? Clinical Immunology and Immunopathology, 69:123-135. 
HOSMER, D. W. \& LEMESHOW, S., 1989. Applied Logistic Regression. New York: John Wiley and Sons.

IBGE (Fundação Instituto Brasileiro de Geografia e Estatística), 1995. Dados obtidos on-line, através do uso do sistema SIDRA II (Sistema IBGE de Recuperação Automática). Dezembro de 1995 \ttp:// www.ibge.gov.br/>.

KLEINBAUM, D.; KUPPER, L. L. \& MORGENSTERN, H., 1982. Epidemiologic Reasearch: Principles and Quantitative Methods. Belmont: Lifetime Learning Publications.

KLEINBAUM, D., 1994. Logistic Regression. A Selflearning Text. New York: Springer-Verlag.

MASSAD, E., 1992. Modelos matemáticos. In: Ecologia, Epidemiologia e Sociedade (O. P. Foratini, ed.), pp. 423-443, São Paulo: Edusp.

MS (Ministério da Saúde), 1989. Controle da Hanseníase, uma Proposta de Integração Ensino-serviço. Rio de Janeiro: Núcleo de Tecnologias Educacionais em Saúde, Divisão Nacional de Dermatologia Sanitária.

MOSIER, D. \& SIEBURG, H., 1994. Macrophage-tropic HIV: Critical for AIDS pathogenesis? Immunology Today, 15:332-339.

NEELOO, S.; AGRAWAL, S. \& RASTOGI, A. K., 1997. Infectious disease and Immunity: Special reference to Major Histocompatibility Complex. Emerging Infectious Diseases, 3:41-49.

NOORDEEN, S. K., 1993. Leprosy 1962-1992. 4. Epidemiology and Control of Leprosy - A review of progress over the last 30 years. Transactions of the Royal Society of Tropical Medicine and Hygiene, 87:515-517

NOWAK, M. A.; ANDERSON, R. M.; MCLEAN, A. R.; WOLFS, T. F. W.; GOUDSMIT, J. \& MAY, R. M., 1991. Antigenic diversity thresholds and the development of AIDS. Science, 254:963-969.
OMS (Organização Mundial da Saúde), 1988. Expert Committee on Leprosy. Sixth Report. Technical Report Series 768. Geneve: OMS.

OMS (Organização Mundial da Saúde), 1998. Leprosy Trend in 32 Endemic Countries. Janeiro de 1998 বttp:// www.who.int/lep/det.htm>.

RANADE, M. G. \&JOSHI, G. Y., 1995. Long-term follow-up of families in an endemic area. Indian Journal of Leprosy, 67:411-425.

RIDLEY, D. S. \& JOPLING, W. H., 1966. Classification of leprosy according to immunity. International Journal of Leprosy, 34:255-273.

SAMPAIO, E. P.; MOREIRA, A. L.; KAPLAN, G.; ALVIM, M. F. S.; DUPPRE, N. C.; MIRANDA, C. F. \& SARNO, E. N., 1991. Mycobacterium leprae-induced Interferon- production by household contacts of Leprosy patients: Association with Development of Active Disease. Journal of Infectious Diseases, 164: 990-993.

STATISTICS AND EPIDEMIOLOGY RESEARCH CORPORATION/CYTEL SOFTWARE CORPORATION, 1994. EGRET (Epidemiological, Graphics, Estimation and Testing). Seattle: Statistics and Epidemiology Research Corporation/Cytel Software Corporation.

VAN BRAKEL, W. H. \& KHAWAS, I. B., 1994. Silent neuropathy in leprosy: An epidemiological description. Leprosy Reviews, 65:350-360.

VAN BEERS, S. M.; IZUMI, S.; MADJID, B.; MAEDA, Y.; DAY, R. \& KLASTER, P. R., 1994. An epidemiological study of leprosy infection by serology and polymerase chain reaction. International Journal of Leprosy and Other Mycobacteria Diseases, 62: 1-9. 